\title{
Kinderkosten und Familiengründung: Erste Befunde einer Prüfung der Neuen Haushaltsökonomie unter Verwendung von Paardaten
}

\author{
Child-related costs and the transition to parenthood: A preliminary test \\ of the New Home Economics using data from German couples.
}

\begin{abstract}
Zusammenfassung:
Am Beispiel der Familiengründung wird die empirische Relevanz der Neuen Haushaltsökonomie (NHE) untersucht. Dabei geht dieser Beitrag in zweierlei Hinsicht über die übliche Anwendung dieses Ansatzes hinaus: Einerseits wird, parallel zur Opportunitätenkostenhypothese, die oft vernachlässigte Einkommenshypothese berücksichtigt. Andererseits wird das in diesem Erklärungszusammenhang zentrale Konzept der Kinderkosten nicht, wie üblich, über bildungs- oder erwerbsbezogene Indikatoren operationalisiert, sondern über die subjektive Einschätzung der persönlichen Relevanz unterschiedlicher Kostenaspekte von Elternschaft. Die Analyse basiert auf Daten von 237 Paaren, die im Rahmen einer Vorstudie des Projektes Panel Analysis of Intimate Relationships and Family Dynamics 2006 erhoben worden sind. Die angesichts des Querschnittscharakters der Daten sowie der eingeschränkten Validität einiger Indikatoren vorläufigen Ergebnisse lassen sich allenfalls als mittelmäßige Bestätigung der NHE interpretieren. Zumindest gewisse Unterstützung erfährt die Opportunitätenkostenhypothese: Mit zunehmender Bildung der Frau steigen die von ihr wahrgenommenen Opportunitätenkosten, was die Wahrscheinlichkeit der Familiengründung herabsetzt. Auch die von der Frau angegebenen direkten Kosten, die mit dem Bildungsniveau ihres Partners sinken, beeinflussen die Erstgeburt negativ, was zunächst für die Einkommenshypothese spricht. Allerdings kann nicht bestätigt werden, dass dieser positive Bildungseffekt seine Wirksamkeit über die
\end{abstract}

\begin{abstract}
:
The aim of this contribution is to examine basic implications of the New Home Economics (NHE) with respect to family formation using data from couples. This investigation extends the multitude of empirical applications of the NHE in two ways: First, the often neglected income hypothesis is tested simultaneously to the prominent opportunity cost hypothesis. Second, child-related costs are measured directly rather than assumed from individual's socio-economic position since they were indicated by the respondent's individual perception. Within a pre-study of the German Panel Analysis of Intimate Relationships and Family Dynamics a sub-sample of 237 couples was conducted in 2006. Due to the cross-sectional nature of the data and the limited validity of some indices the findings are only of preliminary character. They suggest only a moderate confirmation of the NHE. At least, some evidence is provided for the opportunity cost hypothesis. The higher woman's educational level the higher her job related opportunity costs which finally decrease the likelihood of the first birth. No evidence is found with respect to the income hypothesis as man's positive educational effect on family formation seems not to be mediated by the direct childrelated costs perceived by his female partner. Counterevidence is also related to the costs perceived by the male partner as they are not only independent of the educational level but also irrelevant in terms of family formation.
\end{abstract}


Kinderkosten erlangt. Schließlich erweisen sich die von den Männern wahrgenommenen Kinderkosten weder als bildungsabhängig noch haben sie einen Einfluss auf die Familiengründung.

Schlagwörter: Neue Haushaltsökonomie, Familiengründung, Kinderkosten, Paaranalyse
Key words: New Home Economics, family formation, child-related costs, dyadic analysis

\section{Einleitung}

Im Rahmen der Neuen Haushaltsökonomie (NHE) hat Gary S. Becker $(1965,1981)$ paarspezifische Kinderkosten in den Mittelpunkt der Erklärung unterschiedlicher Kinderzahlen gestellt. Wird dieser theoretische Ansatz auf die weithin beobachtbaren rückläufigen Fertilitätsraten angewendet, sowie die vor allem in modernen, westlichen Nationen nachlassende Bereitschaft zur Familiengründung, so werden einerseits die gestiegenen finanziellen Aufwendungen hervorgehoben, die für die Ausstattung und Erziehung der Kinder notwendig sind. Auf der anderen Seite werden erwerbsbezogene Opportunitätenkosten angeführt, die ebenfalls in den letzten Jahrzehnten für weite Teile der Bevölkerung erheblich zugenommen haben, maßgeblich bedingt durch eine allgemeine Anhebung des (beruflichen) Qualifikationsniveaus in Folge der allgemeinen Bildungsexpansion. Die NHE gilt weithin als gut bestätigt, allerdings fußen die meisten empirischen Studien auf zwei nicht unerheblichen Verkürzungen ihrer theoretischen Basis: Erstens wird die Opportunitätenkostenhypothese beinahe nie unter Verwendung direkt erfasster Kosten getestet, vielmehr erfolgt deren Operationalisierung typischerweise über erwerbsbezogene Variablen - allen voran das (Aus-)Bildungsniveau. Dieser Umstand geht nicht zuletzt damit einher, dass in der Umfrageforschung Kinderkosten selten direkt erfragt werden. Eine aussichtsreiche, gleichwohl anspruchsvolle Möglichkeit einer direkten Kostenerfassung besteht in der Abfrage ihrer subjektiven Wahrnehmung. Gewinnbringend könnte ein solches Vorgehen deshalb sein, weil die Messung genau an der Stelle ansetzt, wo die Kosten unmittelbar in den generativen Entscheidungsprozess einfließen. Anspruchsvoll ist es insofern, als der Aussagegehalt der hierüber gewonnenen Daten, stärker als die Erfassung des Bildungsniveaus oder der Berufsposition, hinterfragt werden muss. Ein zweites Defizit vieler Studien besteht darin, dass sie der ebenfalls im Rahmen der NHE formulierten Einkommenshypothese wenig Beachtung schenken. Ob also parallel zum negativen Opportunitätenkosteneffekt, der gemäß der NHE ausschließlich an die Frau geknüpft ist, das Einkommen des Mannes den behaupteten positiven Einfluss auf das generative Verhalten des Paares ausübt, wird empirisch oft vernachlässigt. Das wiederum mag hauptsächlich daran liegen, dass ein simultaner Test beider Hypothesen Daten von beiden Partnern voraussetzt.

Im Rahmen einer Vorstudie des Projektes Panel Analysis of Intimate Relationships and Family Dynamics (PAIRFAM) ${ }^{1}$ wurde nun ein Design verwirklicht, das für beide

1 Dieses von der DFG geförderte Forschungsprogramm wird an vier bundesweiten Standorten unter der Leitung von Josef Brüderl (Universität Mannheim), Johannes Huinink (Universität Bremen), 
Partner die jeweils subjektive Einschätzung der Kinderkosten erhebt. Darüber hinaus liegen für die 237 Paare Angaben zum Bildungsniveau vor, sowie deren retrospektiv erfasste Fertilitätsbiographie. Damit bietet sich die Gelegenheit, zwei Annahmen der NHE empirisch nachzugehen, die einerseits zentral innerhalb ihrer Argumentation sind, andererseits in Analysen typischerweise nicht angemessen berücksichtigt werden. Gleichwohl wird auch die hier präsentierte Untersuchung insofern lückenhaft bleiben, als die durchaus offensichtlichen Vorteile der vorliegenden Daten von nennenswerten Mängeln begleitet werden. So stellt sich die Stichprobe als quantitativ und regional eingeschränkt dar und zentrale Konzepte können nicht optimal abgebildet werden: Die subjektiv wahrgenommenen Kinderkosten werden weitgehend erst nach dem untersuchten generativen Verhalten erfragt und das Bildungsniveau kann lediglich über die schulische Bildung operationalisiert werden. Deshalb ist diese Arbeit nur als erster Versuch zu sehen, die NHE einer kritischeren Prüfung zu unterziehen als bisher geschehen. Insbesondere die empirische Analyse bleibt explorativer Natur, deren Ergebnisse vorläufig sind und unbedingt weiterer Validierung bedürfen. Der Beitrag dieser Arbeit besteht vor allem in der Präsentation und Diskussion von Befunden, die einerseits das Resultat der Überprüfung nicht trivialer Hypothesen sind und die andererseits Anknüpfungspunkt für weiterführende Forschungsarbeiten in dieser Richtung sein können.

Die Untersuchung fokussiert auf den Übergang zur Elternschaft, wofür zwei Gründe ausschlaggebend sind: Erstens ist die Vermutung plausibel, dass ökonomische Kinderkosten bei der Entscheidung für oder gegen ein erstes Kind am stärksten ins Gewicht fallen. Die relativen Preise bzw. Kosten (vor allem die Opportunitätenkosten) pro Kind sollten mit jeder weiteren Geburt abnehmen, weshalb auch ihre Entscheidungsrelevanz tendenziell rückläufig sein sollte. Zweitens begründen aktuelle Trends in Deutschland die Entscheidung für diesen Untersuchungsgegenstand: Angaben zum Anteil kinderloser Frauen in Westdeutschland, die derzeit ihre reproduktive Phase abschließen, verweisen auf circa 25\% (mit mehr oder weniger großen Abweichungen je nach Datengrundlage); entsprechende Werte für Ostdeutschland fallen (derzeit noch) geringer aus (Kreyenfeld/Konietzka 2007). Verrechnet man diesen Wert um die 5 bis $10 \%$, für die in modernen Gesellschaften von einer biologisch verursachten Kinderlosigkeit ausgegangen wird (ibd.: 15), so verbleiben mindestens $15 \%$ Frauen, die mehr oder weniger bewusst auf eigene Kinder verzichten. Das stellt ein durchaus erklärungswürdiges Phänomen dar. Zwar wird dieses empirische Argument durch das hier gewählte Paardesign insofern relativiert, als jüngere Befunde die Bedeutung von veränderten Prozessen der Paarbildung sowie eine sich gewandelte Paarstabilität für die wachsende Kinderlosigkeit herausstellen und andeuten, dass der Übergang zur Elternschaft innerhalb von Partnerschaften kaum abgenommen hat (Duschek/Wirth 2005; Klein 2003). Allerdings findet die hier geplante Untersuchung bereits bestehender Partnerschaften durch das eigentliche Ziel dieses Beitrages Rechtfertigung, nämlich einer empirischen Auseinandersetzung mit der NHE, deren Argumentation explizit auf der Haushalts- bzw. Paarebene angesiedelt ist.

Die Arbeit gliedert sich wie folgt: Das nachstehende Kapitel bietet eine kritische Diskussion der derzeitigen empirischen Befundlage zur Erklärung generativen Verhaltens vor

Bernhard Nauck (Technische Universität Chemnitz) und Sabine Walper (Ludwig-MaximiliansUniversität München) durchgeführt. 
dem Hintergrund der zentralen Argumentationsstränge der NHE. Es schließt mit dem Fazit ihrer bisher unvollständigen Prüfung bzw. Anwendung und formuliert Implikationen für eine adäquate Überprüfung am Beispiel der Familiengründung. Der sich anschließende empirische Teil der Arbeit verfolgt eine erste Annäherung an die Prüfung der abgeleiteten Annahmen. Es werden zunächst die zu Grunde liegenden Daten und Indikatoren vorgestellt, gefolgt von der Präsentation der Ergebnisse sowie deren abschließender Zusammenfassung und Bewertung.

\section{Zum Forschungsstand zur Neuen Haushaltsökonomie: Eine kritische Diskussion}

Bereits vor der stark formalisierten Familienökonomie von Becker (1965) haben ökonomisch motivierte Erklärungsversuche die Kinderkosten als zentrale Erklärungskategorie rückläufiger Geburtenzahlen herausgearbeitet (für eine Übersicht vgl. Bagozzi/van Loo 1978). Dass die Kosten bzw. die Preise von Kindern im Mittelpunkt dieser Erklärungstradition stehen, ist im Wesentlichen der Einbettung in die klassische Preistheorie geschuldet: Individueller Nutzen wird aus Konsumgütern gezogen, deren Erwerb bzw. Produktion mit finanziellen Aufwendungen verbunden ist. Es kann zwischen verschiedenen Gütern, worunter auch Kinder zählen, gewählt werden. Dabei gilt: Je größer das finanzielle Budget ist, desto mehr Güter sind realisierbar und je mehr Güter zur Auswahl stehen, desto höher ist deren Konkurrenz untereinander. Ausgehend von einem solchen Verständnis behauptete bereits Brentano (1910: 381f.) eine im Zuge des gesellschaftlichen Fortschrittes zunehmende, ,intentional limitation in the size of the family, whether the motive be the disinclination of the parents to being hampered in the enjoyment of other pleasures, or their fear of losing the earning capacity which renders these other pleasures accessible“. Auch Leibenstein (1957: 163) thematisierte den negativen Einfluss von direkten und indirekten Kinderkosten auf die Neigung Kinder zu bekommen, wobei die indirekten Kinderkosten vor allem mit Blick auf die Frau wirksam werden. Für sie gilt in noch viel stärkerem Maß als für den Mann: „Opportunities for engaging in productive or in various timeconsuming consumption activities are likely to grow as income increases". Bei Becker erfolgte eine explizite Beschränkung auf ökonomische Kosten. Seine Argumentation bezüglich der angestrebten Realisierung einer maximalen Nutzenproduktion bezieht sich auf den Haushalt bzw. das Paar als Einheit, womit die Zeitallokation beider Partner in den Vordergrund rückt. Die Antwort auf die Frage, welcher Partner sich welchem der beiden Hauptproduktionsbereiche widmet - Erwerbs- oder Haushaltsproduktion - hängt von den jeweils individuellen Produktionsvorteilen ab. Angesichts biologischer und sozialisationsbedingter Vorteile der Frau bei der Hausarbeit, der Geburt und Betreuung von Kindern, setzt diese zumindest den Großteil ihrer Zeit in diesem Bereich ein. Der Mann hingegen geht, begründet durch seine Einkommensvorteile auf dem Arbeitsmarkt, im Idealfall einer Vollzeit-Erwerbstätigkeit nach. Seine Einkommensvorteile ergeben sich einerseits aus seiner im Allgemeinen höheren Berufsbildung. Auf der anderen Seite und unabhängig hiervon resultieren sie aus seinem grundsätzlich höheren Einkommenspotential angesichts realer Einkommensungleichheiten. Gemäß dieser für den Gesamthaushalt vor- 
teilhaften klassischen Arbeitsteilung, ist die Entstehung von Einkommensverlusten an die Frau gekoppelt. Dabei sind diese Opportunitätenkosten von Kindern um so höher, je höher das Einkommen(spotential) der Frau ist und je weniger Zeit sie am Arbeitsmarkt verbringt. „A growth in the earning power of women raises [...] the relative cost of children and thereby reduces the demand for children" (Becker 1981:245).

Als Hinweis für die Richtigkeit dieser Überlegungen ließe sich der Befund anführen, dass in Deutschland vor allem Akademikerinnen überdurchschnittlich häufig kinderlos bleiben. Die Anteile Kinderloser bewegen sich in dieser Qualifikationsgruppe je nach Datengrundlage zwischen 25\% und 40\% (Schaeper 2007: 137). Auch zahlreiche US-amerikanische Studien, die in der Folge der Formulierung der NHE durchgeführt worden sind, konnten negative Einkommens-, (Butz/Ward 1979; Cain/Weininger 1973; de Tray 1973; Hashimoto 1974; Mincer 1963) Erwerbs-, (Conger/Campbell 1978) und Bildungseffekte (Cain/Weininger 1973; Conger/Campbell 1978; Willis 1973) auf die Fertilität nachweisen. Allerdings sind die Ergebnisse nicht durchweg konsistent und beruhen häufig auf der Analyse von Querschnittsdaten oder aggregierten Zeitreihen - Datenmaterial also, das nur eingeschränkt geeignet ist, dem ursächlichen Einfluss individueller Ressourcen auf die Familiengründung nachzugehen. Erst der lebensverlaufsanalytische Zugang unter Verwendung von retrospektiv erhobenen Verlaufsdaten, konnte den negativen Einfluss verlässlich bestätigen, den insbesondere das Bildungsniveau der Frau, jenseits des in diesem Zusammenhang entdeckten Institutioneneffektes (Blossfeld/Huinink 1989, 1991; Blossfeld/Jänichen 1990), auf die Geburt des ersten Kindes ausübt (Brüderl/Klein, 1991, 1993; Klein/Lauterbach 1994; Timm 2006). Entsprechend scheint dieser Argumentationsstrang der NHE empirisch fundiert.

Allerdings wird selten die indirekte Prüfung in Frage gestellt, die Ausbildung, Erwerbstätigkeit und Erwerbseinkommen als Proxy-Indikatoren für die anfallenden Opportunitätenkosten der Kinderbetreuung verwendet. Verschiedene Gründe mögen dieses Vorgehen legitimieren. Becker (1965) selbst hat eine derartige Annäherung an die Opportunitätenkosten vorgeschlagen, ganz in der ökonomischen Tradition der Berufung auf hard facts. Die Erfassung entsprechender Informationen ist einfach zu realisieren und selbst eine retrospektive Abfrage von Bildungs- und Erwerbsbiographien ist vergleichsweise zuverlässig möglich. Tatsächlich scheint sich diese Herangehensweise auch bewährt zu haben, wenn man sich die Befunde der oben genannten Studien vor Augen führt. Trotz dieser offenkundigen Vorteile ist einzuwenden, dass es sich hierbei lediglich um eine indirekte Abbildung der Kinderkosten handelt, die aus der individuellen Verfügbarkeit sozioökonomischer Ressourcen geschlossen werden. Dadurch werden Zusatzannahmen nötig, die selbst nicht direkt überprüfbar sind und das wiederum zieht eine gewisse Uneindeutigkeit bezüglich der behaupteten Mechanismen nach sich. Anhand des am häufigsten zum Einsatz kommenden Indikators der (Aus-)Bildung kann dieses Problem gut verdeutlicht werden: Unterstützt durch inzwischen unzählige Studien in der Tradition des Humankapitalkonzeptes gilt gemeinhin als bestätigt, dass Bildung, vermittelt über die damit einhergehenden Karrierechancen, einen sehr guten Indikator für das Markteinkommen(spotential) abgibt (Brüderl/Klein 1993: 196). Hieran werden (geschlechtsspezifische) Rückschlüsse auf die Kinderkosten geknüpft, von denen schließlich der eigentliche (negative) Einfluss auf das generative Verhalten ausgeht. Aber auch alternative Ansätze zur Erklärung von Fertilität behaupten einen negativen Bildungseffekt, jedoch mit anderen 
Begründungen. Beispielsweise betonen in soziologischer Tradition stehende Modelle eine mit dem Bildungszuwachs einhergehende Modernisierung von Geschlechterrollen und Normvorstellungen. Vermittelt über einen Bedeutungsverlust von Heirat und Familiengründung werden diese für die rückläufige Geburtenneigung verantwortlich gemacht (u.a. Scanzoni 1975; van de Kaa 1987). Indem also die Kinderkosten empirisch nicht direkt abgebildet werden, kann im Sinne der NHE lediglich vermutet werden, dass der etablierte Bildungseffekt seine Wirksamkeit den Kinderkosten verdankt. Eine empirische Trennung zwischen unterschiedlichen Erklärungsfiguren ist auf diese Weise aber nicht möglich. Eine direkte Erfassung der Kinderkosten sollte diesbezüglich mehr Aufschluss bringen.

Bisher gibt es nur wenige Projekte, die dieses Ziel verfolgt haben. Zudem unterscheiden sich die vorliegenden Instrumente sowie die Stichproben, bei denen sie Verwendung gefunden haben, zum Teil erheblich, was deren Ergebnisse nur bedingt vergleichbar macht. Einen frühen Beitrag zur Instrumentenentwicklung hat die Value of ChildrenForschung geleistet (Arnold et al. 1975; Fawcett 1978; Trommsdorff/Nauck 2005). Jüngste Arbeiten, die in diesem Zusammenhang entstanden sind, können auf einigen Erklärungserfolg im internationalen Vergleich (Nauck 2007), sowie bezogen auf die Aufklärung rückläufigen Kinderreichtums (Klaus 2008) zurückblicken. Die Kinderkosten wurden hier über die individuelle Bedeutsamkeit von Gründen erhoben, die gegen die Geburt von (weiteren) Kindern sprechen. Der wesentliche Nachteil dieses Forschungsprogramms besteht allerdings darin, dass die Kinderkosten zeitlich nicht vor dem beobachteten Geburtenverhalten erfasst wurden. Längsschnittliche Daten hält dagegen der Familiensurvey bereit. Eine Auswertung der dort erhobenen negativen Aspekte von Elternschaft bestätigt die NHE nur partiell: Die von Frauen wahrgenommenen Kinderkosten stehen in keiner systematischen Abhängigkeit zu ihrem Bildungsniveau und lediglich von den direkten finanziellen Kinderkosten geht ein negativer Effekt auf die Familiengründungsrate aus. Gänzlich ohne Bedeutung bleiben die Opportunitätenkosten (Klein/Eckhard 2005: 171f.). Angesichts des zu Grunde liegenden Paneldesigns könnte das als durchaus gesicherter Befund gewertet werden. Allerdings stellt sich die Abfrage der Kinderkosten hier eher als Erfassung allgemeiner Ansichten über die Nachteile von Elternschaft dar und weniger als deren subjektive Bewertung vor dem Hintergrund der eigenen Situation. So erlaubt eine starke Zustimmung auf Aussagen wie „Wenn Frauen eine berufliche Karriere wollen, müssen sie auf ihre Kinder verzichten" nicht zwangsläufig den Rückschluss auf eine hohe individuelle Relevanz des jeweils angesprochenen Kostenaspektes. Entsprechend sind die fehlenden Zusammenhänge nicht ohne weiteres als Beleg gegen die NHE zu interpretieren. Ganz anders wurden die Kinderkosten in der in den Niederlanden durchgeführten Längsschnittuntersuchung Panel Study on Social Integration in the Netherlands erfasst. Kinderlose Befragte sollten angeben, wie (stark) die Geburt eines ersten Kindes beispielsweise ihre berufliche Karriere oder ihre finanzielle Situation beeinflussen würde. In einer auf diesen Daten basierenden Analyse wurden die subjektiv eingeschätzten Kinderkosten aber nur für die Erklärung des Zeitpunktes der Erstgeburt eingesetzt mit dem Befund: „The higher the perceived costs of childbearing for people's career's, the longer first childbirth is postponed" (Liefbroer 2005: 387). Ob und welcher Einfluss von ihnen auf das in der NHE zentrale Geburtenrisiko ausgeht, bleibt damit leider offen. Eine Zwischenbilanz zur Validität und zum möglichen Erkenntnisgewinn einer direkten Kostenabfrage lässt sich in Anbetracht der Heterogenität dieser Studien nur schwer ziehen. Ge- 
meinsam ist ihnen allerdings, dass sie ausschließlich individuelle Entscheidungsprozesse betrachten.

Das verweist auf das zweite Defizit, das viele empirische Studien zu diesem Thema kennzeichnet: Sie tragen der Prämisse keine Rechnung, dass das Paar und nicht der Einzelne Entscheidungsträger ist. Zwar kann aus verhandlungstheoretischer Sicht diese unterstellte Haushaltsrationalität durchaus in Frage gestellt werden (Ott 1989); geht es aber zunächst darum, sie einer adäquaten Überprüfung zu unterziehen, verlangt das die Berücksichtigung der in diesem Zusammenhang formulierten zweiten Hypothese, die explizit auf die direkten Kinderkosten abhebt. Die entsprechende Hypothese hierzu formuliert einen positiven Einfluss des vom Mann erzielten Einkommens auf die Fertilität und auch sie ist an die Annahme gekoppelt, dass der Mann ausschließlich für die Marktproduktion zuständig ist. Mit wachsendem Einkommen verbessern sich die Möglichkeiten des Erwerbs von Marktgütern, die ebenfalls für die Produktion von Kindern notwendig sind. Entsprechend ist ein positiver Einkommenseffekt auf die Familiengründung zu ergänzen. Zwar verweisen viele Beiträge mit großer Regelmäßigkeit auf diese Ableitung der NHE, mit beinahe ebensolcher Regelmäßigkeit beschränken sie sich dann aber auf die Überprüfung der Opportunitätenkostenhypothese. Das geht damit einher, dass im Mittelpunkt vieler Studien zur Fertilität ausnahmslos Frauen stehen. ${ }^{2}$ Obwohl in jüngster Zeit Arbeiten entstanden sind, die sich diesem Thema (auch) aus männlicher Perspektive nähern (u.a. Eckhard/Klein 2007), sind Paaranalysen nach wie vor sehr rar. Solange sozio-ökonomische Indikatoren Verwendung finden sollen, sind die hierfür erforderlichen Paarinformationen einfach zugänglich: Entsprechende Partnerdaten können recht valide über die eigentliche Befragungsperson gewonnen werden, was auch ein inzwischen übliches Vorgehen darstellt. Hingegen kann auf die Befragung beider Partner nicht verzichtet werden, wenn subjektiv wahrgenommene Kinderkosten erfasst werden sollen und das wiederum verlangt ein sehr aufwendiges Befragungsdesign.

So verwundert es nicht, dass ein Wechsel von der Individual- zur Paaranalyse generativen Verhaltens empirisch bisher nur selten vollzogen wurde, dabei liefern die wenigen, hierzu existierenden Beispiele, weiterführende Erkenntnisse (Klein 2003; Kurz 2005; Wirth 2007). Beispielsweise finden sich bei Wirth (2007: 188) empirische Belege, die für die NHE sprechen: „Die geringste Neigung zur Familiengründung zeigt sich [...] bei einem formalen Bildungsvorsprung der Partnerin, d.h. den hypogamen Paaren. [Und das ist] vor allem dann zu beobachten, wenn der Partner zwar einen allgemein bildenden Schulabschluss, aber keinen beruflichen Ausbildungsabschluss [...] aufweist“. Das bestätigt die NHE, da dieser Konstellation sehr hohe, durch die hohe Bildung der Frau verursachte Opportunitätenkosten unterstellt werden können, bei gleichzeitig hohen direkten Kinderkosten angesichts des geringen Einkommens(potentials) des Mannes. Eine paarbezogene Auswertung des Familiensurveys liefert ebenfalls Hinweise, die die NHE stützen, indem sie einen an die Frau gekoppelten negativen Opportunitätenkosteneffekt herausgestellt und parallel dazu einen positiven Einkommenseffekt des Mannes aufdeckt (Klein 2003: 518). Der maßgebliche Nachteil dieser Beiträge besteht jedoch darin, dass die Kin-

2 Erst jüngere, international etablierte demographische Surveys wie der Generations and Gender Survey beziehen auch systematisch Männer in die Datenerhebung ein, während ältere Großprojekte wie der Demographic and Health Survey ausschließlich Frauen befragen. 
derkosten über das Bildungsniveau operationalisiert werden. Vergleichbare Arbeiten unter Verwendung subjektiv eingeschätzter Kinderkosten liegen meines Wissens nicht vor.

Soweit steht also eine adäquate Anwendung der NHE im Bereich des generativen Verhaltens aus. Als Schlussfolgerung aus der hier gesammelten Kritik müsste die zwischen Bildung und Geburtenverhalten vermittelnde Funktion der wahrgenommenen Kinderkosten im Rahmen einer Paaruntersuchung getestet werden. Wie einleitend vorweggenommen und im folgenden Kapitel näher ausgeführt, lässt sich auch mit den vorliegenden Daten keine Prüfung vornehmen, die alle soeben dargelegten Defizite gleichzeitig behebt. Mindestens aber kann eine erste empirische Annäherung an eine Analyse stattfinden, die insbesondere den beiden Hauptkritikpunkten der vorangegangenen Betrachtung Rechnung trägt. Es soll also in einem ersten Schritt geprüft werden, ob die wahrgenommenen Opportunitätenkosten mit Zunahme des Bildungsniveaus der Frau steigen, während die direkten Kosten mit der Bildung des Mannes abnehmen. Anschließend ist zu testen, ob ein Zuwachs an wahrgenommenen Kinderkosten eine rückläufige Neigung des Paares zur Familiengründung nach sich zieht und inwiefern den Kinderkosten hierbei tatsächlich eine vermittelnde Funktion zwischen Bildung und Familiengründung zukommt.

\section{Daten und Methode}

\section{Daten}

Eine 2006 realisierte Teilstichprobe der PAIRFAM-Vorstudie findet für die folgenden Analysen Verwendung. Da in Ergänzung zur eigentlichen Befragungsperson auch deren Partner befragt wurde, konnte ein Teildatensatz heterosexueller Partnerschaften gewonnen werden. Die Stichprobe ist räumlich auf die vier Großstädte Bremen, Chemnitz, Mannheim und München beschränkt, innerhalb derer jeweils Zufallsstichproben von Befragten ausgewählter Geburtskohorten aus den städtischen Melderegistern gezogen wurden. In Anlehnung am bundesweiten durchschnittlichen Erstgebäralter wurden aus der Analyse Fälle ausgeschlossen, in denen mindestens einer der Partner nach 1980 geboren wurde sowie Partnerschaften, die erst im Beobachtungsjahr eingegangen wurden. Ferner fanden nur solche Paare Eingang in die Analysen, deren erstes Kind ein gemeinsames leibliches Kind ist. Für andere Formen der Familiengründung werden spezifische Entscheidungsmuster vermutet. So ist beispielsweise im Fall von Stiefkindern anzunehmen, dass die hier zur Prüfung stehenden Mechanismen insbesondere durch paarbildungsrelevante Entscheidungskriterien überlagert werden, weil die Entscheidung für ein Stiefkind im Allgemeinen an die Entscheidung für eine Partnerschaft gekoppelt ist. Es verbleiben 237 Paare, die die Geburtsjahre zwischen 1928 und 1980 abdecken sowie unterschiedliche Kinderzahlen, die innerhalb der betrachteten Partnerschaften realisiert worden sind: So sind 14\% der Partnerschaften (noch) kinderlos, 25\% haben ein Kind, 61\% zwei Kinder oder mehr. Es wird deutlich, dass es sich um eine kleine und sehr selektive Stichprobe handelt, was allerdings einer Prüfung von Zusammenhangshypothesen insbesondere deshalb nicht grundsätzlich entgegen steht, weil die von der NHE behaupteten Thesen nicht auf bestimmte Bevölkerungssegmente beschränkt sind. Von einigem Nachteil ist hingegen der Querschnittscharakter der Erhebung: Ein Großteil der Paare hat den hier interes- 
sierenden Übergang in die Elternschaft zum Messzeitpunkt bereits vollzogen. Zwar wurde dieser Zeitpunkt retrospektiv erfasst, Gleiches war jedoch nicht für die subjektive Kostenwahrnehmung möglich. Deshalb liegen die Kinderkosten für einen Teil der Paare erst nach der Erstgeburt vor, was zur Infragestellung ihrer Validität und der daran geknüpften Kausalzusammenhänge berechtigt. Gewisse nachträgliche Anpassungen der eingeschätzten Kinderkosten, etwa an das gezeigte Verhalten, an zwischenzeitlich gemachte Erfahrungen im Verlauf der eigenen Elternschaft oder sich gewandelte Rahmenbedingungen, sind ebenso wenig auszuschließen wie Alterseffekte. Auch wenn keine systematischen Verzerrungen in die eine oder andere Richtung erwartet werden, muss die Möglichkeit ihres grundsätzlichen Auftretens mindestens bei der Beurteilung der Ergebnisse Berücksichtigung finden.

\section{Bildungsniveau}

Das Bildungsniveau ist ein häufig zur Prüfung der NHE eingesetzter Indikator der Kinderkosten. Begründet durch eine enge Koppelung zwischen beruflichem Abschluss und dem Einkommen(spotential), kommt oft ein aus Schul- und Berufsbildung kombinierter Index zur Anwendung (Blossfeld/Huinink 1989; Blossfeld/Jänichen 1990; Brüderl/Klein 1993; Klein/Lauterbach 1994). Einer unzureichenden Erfassung des Berufsabschlusses geschuldet, der nicht für beide Partner vorliegt, muss Bildung in dieser Analyse über den höchsten erreichten Schulbildungsabschluss operationalisiert werden. Dieser bietet zumindest den Vorteil, dass er im Allgemeinen früh im Lebenslauf und typischerweise vor der Familiengründung erreicht wird, um anschließend weitgehend unverändert zu bleiben. Stellt man in Rechnung, dass alle Befragten 26 Jahre und älter sind und bereits über mindestens einen ersten allgemeinbildenden Schulabschluss verfügen, scheint eine empirische Modellierung des impliziten Kausalzusammenhangs zwischen Einkommen(spotential) und wahrgenommenen Kosten zulässig. Der Index weist vier Ausprägungen auf: Hauptschulabschluss, Realschulabschluss, Fachhochschulreife, Hochschulreife. Nur sehr wenige Befragte verfügen über keinen oder einen alternativen Abschluss. Diese Fälle gehen nicht in die Analysen ein. Die Verteilung zeigt, dass Befragte mit hohem Schulbildungsniveau, d.h. jenseits der Realschule, überproportional vertreten sind. Das trifft sowohl für Frauen (47\%) als auch für Männer (52\%) zu. Da das Einkommen(spotential) vor allem ab dem höchsten Bildungssegment deutlich anwachsen sollte, dürfte die Überrepräsentation dieser Befragten eher von Vorteil für die Zusammenhangsprüfungen sein.

\section{Kosten von Kindern}

In deutlicher Abgrenzung von der Konzeption im Familiensurvey, zielte das hier eingesetzte Messinstrument auf keine allgemeine Erfassung der Nachteile ab, die typischerweise mit Kindern einhergehen. Vielmehr stand die Wahrnehmung der eigenen Betroffenheit hiervon im Vordergrund. In Anlehnung an das Instrumentarium der Value of ChildrenReplikationsstudie (Trommsdorff/Nauck 2005), wurde über eine Liste von Items die individuelle Relevanz verschiedener Kostenaspekte von Kindern erfragt. Unter Verwendung einer fünfstufigen Antwortskala, die von sehr unproblematisch (Wert 1) bis sehr proble- 
matisch (Wert 5) reicht, sollte erfasst werden, wie stark vor dem Hintergrund der jeweils persönlichen Lebenslage, verschiedene negative Folgen von Elternschaft erwartet und wie problematisch sie für die jeweils angesprochenen Lebensbereiche eingeschätzt werden. Neben dem bereits diskutierten Nachteil, dass die Kosten nicht durchweg vor der Familiengründung erfragt wurden, sieht sich diese Art der Operationalisierung von Kinderkosten vor zwei weitere Herausforderungen gestellt: Erstens unterliegt die Messung einem höheren Risiko, von der Wirksamkeit unterschiedlicher Arten von Antworttendenzen betroffen zu sein. Zweitens bietet ihre Auswertung mehr Raum für die Interpretation dessen, was damit erfasst wird. Somit ist eine sorgfältige (Konstrukt-)Validierung entscheidend, um ein Mindestmaß an Zuverlässigkeit der Indikatoren zu gewährleisten. Mit diesem Ziel wurden zunächst die Kostenitems mit akzeptablen Verteilungen ausgewählt, um sie anschließend einer umfassenden Strukturprüfung zu unterziehen: Im Verlauf einer Reihe iterativer (schiefwinkliger) Faktoranalysen wurden weitere Items ausgeschlossen, sofern sie sich keiner der Kostendimensionen eindeutig zuordnen ließen. Dieses für Männer und Frauen getrennt durchgeführte Verfahren erbrachte für beide Geschlechter dieselbe Minimallösung, die die Grundlage der Indikatorbildung darstellt. Demnach werden die Opportunitätenkosten über einen Mittelwertsindex, bestehend aus den folgenden Items, abgebildet: Wie problematisch ist es für Sie ganz persönlich, (i) dass es schwierig ist Beruf und Familie zu vereinbaren; (ii) dass man wegen Kindern seinen Arbeitsplatz verlieren kann; (iii) dass man wegen Kindern seine beruflichen Ziele nicht erreichen kann? Die interne Konsistenz erweist sich mit Alpha-Koeffizienten von 0,73 (Frauen) und 0,62 (Männer) als gut. Die direkten Kosten ergeben sich aus den beiden Items: (i) dass man sich mit Kindern weniger leisten kann und (ii) dass durch Kinder weniger Geld für die eigene Altersvorsorge bleibt. Die Korrelationen fallen mit $\mathrm{r}=0,74$ (Frauen) und $\mathrm{r}=0,83$ (Männer) hoch aus. Dass für Männer und Frauen vergleichbare Skalen mittlerer Qualität aufgefunden wurden, spricht für eine gewisse Validität der Messung.

\section{Familiengründung}

Von beiden Partnern wurden retrospektiv die Fertilitätsbiographien erfasst, so dass die Information zur Realisierung und zum Zeitpunkt der Geburt des ersten Kindes vorliegt. Angesichts nicht durchweg monatsgenauer Erfassung von Geburtsdaten muss sich auf jahresgenaue Informationen beschränkt werden. Da sich ein Teil der Paare noch in der reproduktiven Phase befindet, stellt sich die abhängige Variable entsprechend als rechtszensiert dar, weshalb ein ereignisanalytisches Verfahren zum Einsatz kommt. Der Übergang zum ersten Kind wird mittels einer modifizierten Version des log-logistischen Modells geschätzt (Brüderl/Diekmann 1995), dessen entscheidender Vorteil in einer Trennung unterschiedlicher Parameter des betrachteten Übergangs besteht. Diese Trennung ermöglicht die separate Schätzung potentieller Effekte der Kinderkosten auf drei Ausprägungen, die den Übergang zum ersten Kind beschreiben: Intensität, Lage auf der Zeitachse, sowie Form. Da die hier auf dem Prüfstein stehenden Thesen der NHE auf das Auftreten der Geburt abzielen (und weniger auf ihren Zeitpunkt), wird der Parameter geschätzt, der auf das Risiko einer Erstgeburt verweist (= Intensität). 


\section{Befunde}

\section{Kinderkosten aus Paarperspektive}

Betrachtet man sich zunächst die Höhe der von den Befragten wahrgenommenen Kinderkosten, so zeigt sich, dass sie recht gering ausfallen, da die Mittelwerte beider Kostenaspekte überwiegend in der unteren Hälfte der Skala rangieren. Eine für Eltern und kinderlose Paare getrennte Darstellung (Abbildung 1) weist für die Paare die geringsten Kosten aus, die sich bereits für das erste Kind entschieden haben. Bei den (noch) Kinderlosen sind es vor allem die Opportunitätenkosten, die sie deutlich höher bewerten und das trifft hauptsächlich auf die Einschätzungen der Frauen zu. Geschlechterunterschiede zeigen sich vor allem für (noch) kinderlose Befragte und zwar dergestalt, dass Frauen deutlich höhere Mittelwerte aufweisen als Männer. Geht man davon aus, dass diese Befunde nicht ausschließlich dadurch hervorgerufen werden, dass Kinderkosten systematisch abgewertet werden, nachdem sich ein Paar für die Elternschaft entschieden hat - etwa um die Entstehung von Dissonanz zu vermeiden - dann können diese Gruppenvergleiche durchaus im Sinne der NHE ausgelegt werden: Eine hohe Einstufung der Kinderkosten verhindert tendenziell die Familiengründung. Angesichts der Rechtszensierung der Familiengründung ist sogar von einer leichten Unterschätzung der Gruppenunterschiede auszugehen. Gegen die NHE würde jedoch sprechen, dass sich kinderlose Männer und solche, die bereits Kinder haben kaum in ihren Kostenangaben unterscheiden. Damit deutet sich ein scheinbar geringer Zusammenhang zwischen der Fertilität des Paares und den von Männern wahrgenommen Kinderkosten an, der durch spätere Analysen bestätigt werden wird. Gleichzeitig schwächt diese Tendenz den Verdacht einer allgemeinen Abwertung der Kosten infolge der Geburt des ersten Kindes.

Abbildung 1: Gruppenspezifische mittlere Kinderkosten

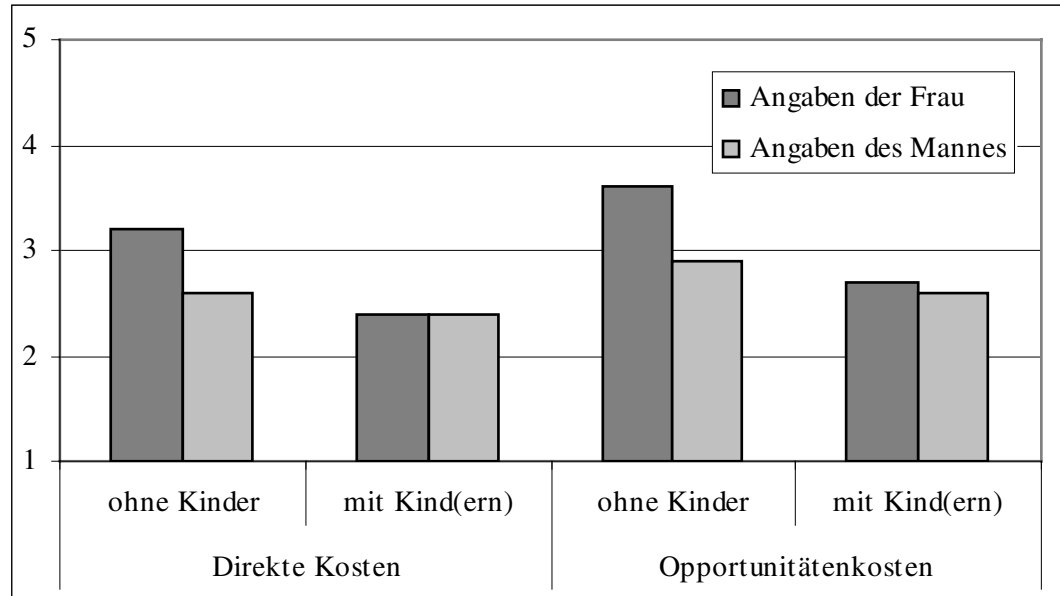

Quelle: PAIRFAM Vorstudie 2006.

Diese präsentierten Mittelwerte werden um eine Betrachtung auf der Paarebene ergänzt, die nunmehr das Verhältnis zwischen konkreten Partnern aufdeckt: In Tabelle 1 sind hier- 
zu die Verteilungen der Partner auf drei Gruppen partnerschaftlicher (Nicht-)Übereinstimmung ausgewiesen. Für die Zuordnung wurde die Differenz der jeweiligen Kostenindikatoren beider Partner berechnet, wobei das Paar als homogen eingestuft wurde, wenn sich die Differenz im Bereich der Standardabweichung bewegt. Insgesamt belegen die Befunde hohe Übereinstimmungen auf Paarebene, da die Kostenangaben beider Partner bei etwa zwei Drittel der Paare auf ähnlichem Niveau rangieren. Insbesondere die Übereinstimmung hinsichtlich der direkten finanziellen Kinderkosten kann als Indiz für die Richtigkeit der von der NHE behaupteten Haushaltsnutzenfunktion verstanden werden: Der Einschätzung der mit Kindern verbundenen finanziellen Aufwendungen scheint das gemeinsame Haushaltsbudget beider Partner zu Grunde zu liegen.

Tabelle 1: Ausmaß partnerschaftlicher Übereinstimmung der wahrgenommenen Kinderkosten

\begin{tabular}{lccc|ccc}
\hline & \multicolumn{3}{c}{ Prozentuale Anteile } \\
Relative Kosten & Alle & $\begin{array}{c}\text { Direkte Kosten } \\
\text { Paare ohne }\end{array}$ & $\begin{array}{c}\text { Opportunitätenkosten } \\
\text { Pare mit }\end{array}$ & \multicolumn{2}{c}{$\begin{array}{c}\text { Alle } \\
\text { Paare ohne }\end{array}$} & $\begin{array}{c}\text { Paare mit } \\
\text { Kinder }\end{array}$ \\
& Paare & Kinder & Kind(ern) & Paare & Kindern \\
\hline Mann höher & 16 & 12 & 16 & 11 & 6 & 12 \\
Homogen & 65 & 53 & 67 & 69 & 62 & 71 \\
Frau höher & 20 & 35 & 17 & 20 & 32 & 17 \\
\hline N & 232 & 34 & 198 & 231 & 34 & 197 \\
\hline
\end{tabular}

Quelle: PAIRFAM Vorstudie 2006.

Daneben bestätigt sich der Befund der Aggregatbetrachtung insofern, als auch innerhalb konkreter Partnerschaften Frauen tendenziell mehr Kosten angeben als Männer. Die Tabelle 1 weist höhere Anteile der Paare aus, in denen die von der Frau wahrgenommenen Kosten über denen ihres Mannes liegen (20\%), als Paare mit umgekehrtem Verhältnis (direkte Kosten $=16 \%$ bzw. Opportunitätenkosten $=11 \%$ ). Betrachtet man ausschließlich (noch) kinderlose Paare, so verstärkt sich diese Geschlechterdifferenz erheblich. Wie ist diese Beobachtung zu deuten? Dass sich das Ausmaß der Übereinstimmung zwischen Paaren mit und ohne Kind(ern) so offensichtlich und systematisch unterscheidet, lässt sich mit der NHE nicht in Einklang bringen. Vor allem die direkten Kinderkosten sollten von beiden Partnern ähnlich hoch wahrgenommen werden und zwar unabhängig von der Familienphase. Geht man also von annähernd validen Kostenmessungen aus, dann entkräftet dieser Befund die eben noch unterstützte Haushaltsnutzenfunktion. Jenseits der NHE lässt sich vermuten, dass neben der absoluten Höhe der Kosten, eine diesbezüglich nicht zwangsläufig gegebene Einigkeit beider Partner förderlich für den Übergang zur Elternschaft ist. Diese Überlegung ist durchaus plausibel, muss jedoch verworfen werden, da spätere (nicht abgedruckte) Analysen zeigen, dass der Übergang zur Elternschaft in keinem bedeutsamen Zusammenhang mit der (fehlenden) Homogamie der Kostenwahrnehmungen steht. Entsprechend wird auch eine zweite Alternativerklärung entkräftet, die an der Querschnittserfassung der Kinderkosten ansetzt und wonach die Wahrscheinlichkeit der Übereinstimmung beider Partner insbesondere mit der gemeinsamen Erfahrung mit eigenen Kindern steigt, da die tatsächlich auftretenden Kinderkosten nunmehr besser eingeschätzt werden können. Erklärungsbedürftig wäre bei einer solchen Auslegung ohne- 
hin, warum dann vor allem Frauen ihre Kostenwahrnehmungen nach unten, hin zu ihren Partnern anpassen. Als Bilanz dieser deskriptiven Betrachtung ist eine, vor dem Hintergrund der NHE sehr gemischte Befundlage festzuhalten.

\section{Bildungsniveau und Kinderkosten}

Im nächsten Schritt wird untersucht, ob das Bildungsniveau beider Partner die wahrgenommenen Kinderkosten so abbildet, wie von der NHE behauptet. Hierzu wurde die dyadische Datenstruktur dem actor-partner interdependence model folgend analysiert (Kashy/Kenny 2000) - ein Vorgehen, das mögliche Abhängigkeiten zwischen beiden Partnern explizit berücksichtigt bzw. testet. Getrennt für beide Kostendimensionen wurden dyadische Pfadmodelle berechnet, die individuelle sowie Partnereffekte der Bildung auf die von beiden Partnern wahrgenommenen Kosten simultan überprüfen. Die Ergebnisse beider Modelle sind in der Abbildung 2 zusammengestellt, wobei standardisierte Beta-Koeffizienten bzw. Korrelationskoeffizienten sowie die zugehörigen Signifikanzen berichtet werden.

Zunächst soll das Modell zur Vorhersage der Opportunitätenkosten betrachtet werden (kursiv gekennzeichnet). Wie der NHE folgend zu erwarten ist, steigen die von der Frau wahrgenommenen Opportunitätenkosten mit der Höhe ihres formalen Schulabschlusses an. Allerdings ist der Koeffizient von sehr geringer Stärke (Beta $=0,23 ; \mathrm{p} \leq 0,01)$, wenn man bedenkt, dass die NHE das Bildungsniveau als geeignetes Proxy für die Kinderkosten versteht. Möglicherweise wird dieser Zusammenhang angesichts der dahinter stehenden und durchaus strittigen Zusatzannahme der Unvereinbarkeit von Erwerbs- und Familienarbeit unterschätzt. Diese Annahme ignoriert kontextspezifische Handlungsbedingungen, was insbesondere bei gesonderter Betrachtung von Ost- und Westdeutschland nicht haltbar scheint: Nach wie vor gestalten sich die Gelegenheitsstrukturen für institutionelle Kinderbetreuung in Ostdeutschland deutlich günstiger und entsprechend sollte der $\mathrm{Zu}$ sammenhang zwischen Bildung und Opportunitätenkosten hier geringer ausfallen als in Westdeutschland, wo die Unvereinbarkeitsannahme besser greift. Dieser Überlegung konnte durch eine getrennte Berechnung des Modells der Abbildung 2 empirisch nachgegangen werden, erbrachte aber überraschenderweise eine leichte Tendenz entgegen der soeben formulierten Erwartung (Gesamtmodell nicht abgebildet): Der Effekt der Bildung der Frau auf die von ihr angegebenen Opportunitätenkosten liegt in Ostdeutschland (Beta $=0,30 ; p \leq 0,05)$ etwas über dem in der westdeutschen Teilstichprobe (Beta $=0,20 ; p \leq 0,05$ ). Es mag sich nun die Vermutung aufdrängen, dass gerade die in Ostdeutschland in den letzten Jahren vorherrschende prekäre Arbeitsmarktlage, die insbesondere die Konkurrenzfähigkeit von Frauen mit Kind(ern) erheblich beeinträchtigt, vor allem bei höher gebildeten Frauen einen allgemeinen Anstieg der mit Kindern in Verbindung gebrachten Opportunitätenkosten bewirkt hat, also auch bei den Frauen, deren Familiengründung bereits einige Zeit zurückliegt. Das impliziert für ostdeutsche Frauen eine nachträgliche Anpassung der Kinderkosten an die sich veränderten ökonomischen Rahmenbedingungen, was sich konkret in einer Zunahme der von ihnen angegebenen Opportunitätenkosten äußern sollte. Diese Interpretation würde die Messung der Kinderkosten empfindlich in Frage stellen. Gegen sie sprechen allerdings zwei Befunde: Erstens fallen die um Geburtsjahr 
und Bildungsniveau bereinigten Mittelwerte der von den ostdeutschen Frauen wahrgenommenen Opportunitätenkosten signifikant geringer aus, als die der in Westdeutschland lebenden Frauen ( $M W=2,7$ gegenüber 3,0; $\leq \leq 0,05$ ). Zweitens legen (hier nicht abgedruckte) Analysen für Ostdeutschland keine schlechtere Vorhersage der Familiengründung durch die Opportunitätenkosten nahe als für Westdeutschland, vielmehr zeichnet sich eine geringfügige Tendenz entgegengesetzter Richtung ab. Angesichts unterschiedlicher Stichproben(größen) ist ein direkter Vergleich der Höhe der Koeffizienten aber ohnehin nicht empfehlenswert. Verlässlicher ist es, sich auf einen Vergleich der Signifikanzen zu beschränken und demnach verweisen die Koeffizienten beider Teile Deutschlands auf eine vergleichbare Erklärungsrelevanz. Im Prinzip sollte das auch bei der Bewertung der regionenspezifischen Zusammenhänge zwischen Bildung und Opportunitätenkosten bedacht werden und so gesehen unterscheiden sich auch diese Koeffizienten in beiden Teilstichproben nur marginal. Was allerdings bleibt ist ihre geringe Höhe. Ob aber angesichts des unbefriedigenden Indikators für das Bildungsniveau hieraus eine eingeschränkte Brauchbarkeit der Abbildung weiblicher Opportunitätenkosten über das Bildungsniveau geschlossen werden kann, ist hier nicht abschließend zu entscheiden. Zumindest erbringt diese Analyse ein Indiz gegen diese übliche Praxis.

Abbildung 2: Individuelle und Partnereffekte des Bildungsniveaus auf die Kosten von Kindern

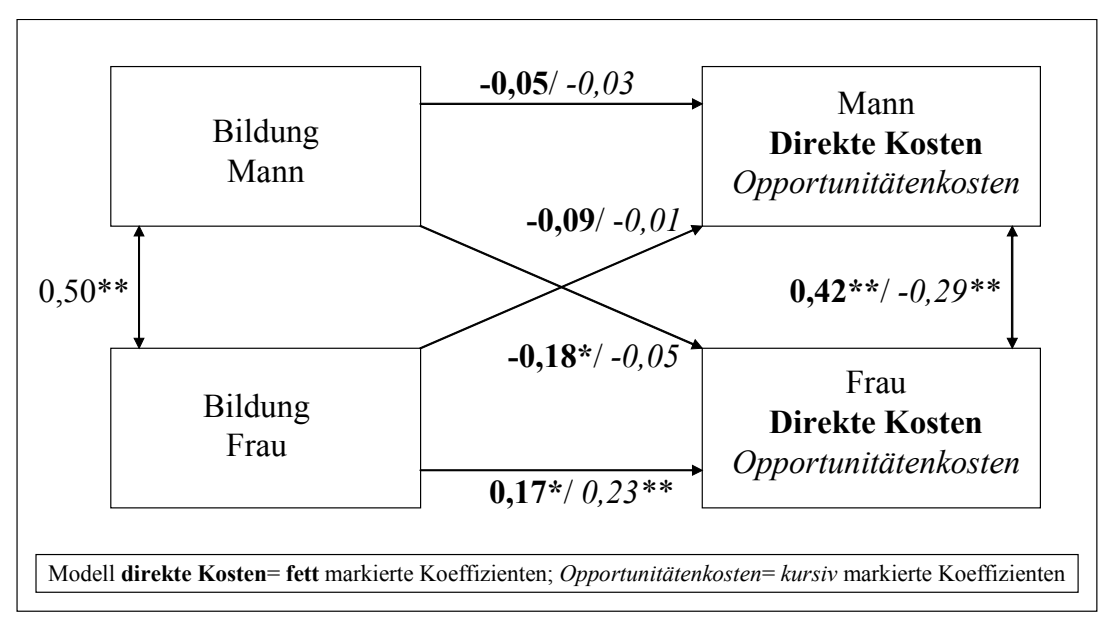

Quelle: PAIRFAM Vorstudie 2006. Anmerkung: Simultane Prüfung der Bildungseffekte von Frau und Mann; $\mathrm{N}=216 ;{ }^{*} \mathrm{p} \leq 0,05 ;{ }^{* *} \mathrm{p} \leq 0,01$.

Dieser Eindruck wird durch das Modell der direkten Kinderkosten verstärkt (fett gekennzeichnet). Es zeigt zunächst einen signifikanten Partnereffekt für die direkten Kosten: Je höher das Bildungsniveau des Mannes ist, desto geringer fallen die direkten Kinderkosten aus, die dessen Partnerin wahrnimmt (Beta $=-0,18 ; \mathrm{p} \leq 0,05)$. Dieser Zusammenhang stützt die Annahme der NHE, wonach die für Kinder notwendigen monetären Ausgaben vor dem Hintergrund einer guten Einkommenssituation des Haushaltes, die im Wesentlichen 
von der Erwerbstätigkeit des Mannes geprägt wird, weniger negativ wahrgenommen werden. Allerdings fällt auch hier - ähnlich dem soeben diskutierten Zusammenhang - der Koeffizient sehr gering aus, was nun einerseits wiederum als Unterschätzung infolge einer eingeschränkten Validität der Indikatoren verstanden werden kann. Andererseits können erneut Zweifel formuliert werden, ob sich über das Bildungsniveau tatsächlich in ausreichendem Maße Rückschlüsse auf die Kinderkosten ziehen lassen. Für empirische Analysen scheint es zumindest empfehlenswert weitere Merkmale einzubeziehen, die ebenfalls auf das erwerbsbezogene Humankapital verweisen und die im Allgemeinen auch in der ökonomischen Theorie thematisiert werden wie Umfang und Dauer der Erwerbstätigkeit, Berufserfahrung und die berufliche Stellung.

Zwei weitere Pfadkoeffizienten dieses Modells deuten eine gewisse Unvollständigkeit der NHE an: Erstens bleiben die seitens des Mannes angegebenen direkten Kosten von dessen Bildung weitgehend unberührt. Das widerspricht der Haushaltsbetrachtung, wonach die finanziellen Ressourcen beider Partner im Gesamthaushaltsbudget zusammenfließen und vor diesem Hintergrund die Wahrnehmungen beider Partner gleichermaßen beeinflussen. Zweitens steigen die von der Frau wahrgenommenen direkten Kosten mit ihrem eigenen Bildungsniveau an (Beta $=0,17 ; \mathrm{p} \leq 0,05)$. Vereinbar mit der NHE wäre allenfalls ein gemäß der Einkommenshypothese negativer Zusammenhang, wonach ein mit hoher Bildung einhergehendes hohes Einkommenspotential der Frau zumindest über eine Teilzeitbeschäftigung realisierbar ist und auf diese Weise ebenfalls zu einer Erhöhung des Haushaltsbudgets beiträgt. Dass dieser Effekt jedoch positiv ausfällt, könnte darauf hinweisen, dass mit steigendem Bildungsniveau der Frau auch ihr Anspruch an die Qualität der Kinder wächst, was angesichts eines höheren Investitionsbedarfes in deren Betreuung und Ausbildung wiederum den Preis pro Kind erhöht und damit auch die antizipierten Kinderkosten. Ein solcher Effekt wird in ähnlicher Weise auch von Becker diskutiert (Becker/Lewis 1973: 281), aber möglicherweise unterschätzt oder somit in der weiteren Argumentation nicht angemessen berücksichtigt. Sofern dieser Mechanismus tatsächlich zum Tragen kommt, wäre er aber auch für Männer erwartbar. Und vielleicht kommt er auch dort zur Wirkung, überlagert allerdings den eigentlich erwarteten negativen Effekt, woraus der gefundene Koeffizient nahe Null $($ Beta $=-0,05)$ resultiert. Diese Befunde geben zu bedenken, Überlegungen aufzunehmen, die bereits in andere ökonomische Modelle Eingang gefunden haben (u.a. Leibenstein 1974: 472f.) und wonach an die Zugehörigkeit zu einer bestimmten Bildungs- und Einkommensschicht spezifische normative Erwartungen hinsichtlich der in Kinder zu tätigende Investitionen gekoppelt sind. In diesem Sinne kritisierte bereits Turchi (1975: 113), dass die NHE derlei „normative factors which may influence the cost of a child as perceived by prospective parents" völlig außer Acht lässt. $\mathrm{Ob}$ nun die hier gefundenen Effekte wiederum dadurch forciert sind, dass der Bildungsindikator auf dem Schulabschluss basiert und die berufliche Bildung nicht einbezieht, muss erneut zu bedenken gegeben werden. Mindestens aber regen die Befunde an, die Notwendigkeit einer solchen Ergänzung der NHE in Betracht zu ziehen. 


\section{Verhaltensrelevanz von Bildungsniveau und Kinderkosten}

Abschließend wurden die von beiden Partnern wahrgenommenen Kinderkosten auf ihre Erklärungskraft hinsichtlich des Übergangs zur Elternschaft untersucht. In Tabelle 2 sind die Übergangsraten zusammengestellt, wobei verschiedene Modelle berechnet wurden: Zunächst finden ausschließlich die geschlechtsspezifischen Kinderkosten Beachtung (Modell 1). Diese werden in den folgenden Modellen durch das Bildungsniveau ergänzt, um der Frage nachzugehen, ob ein primärer Einfluss des Bildungsniveaus auf die Fertilität tatsächlich über die Kosten vermittelt wird. Hierzu wird einem Vorgehen gefolgt, dass Baron/Kenny (1996) zur Ermittlung von Mediatoreffekten vorgestellt haben: Nachdem die vorangegangenen Analysen bereits moderate Zusammenhänge zwischen Bildung und Kinderkosten belegt haben, ist nunmehr zu prüfen, ob erwartungskonforme Primäreffekte der Bildung auf die Familiengründung bestehen (Modelle 2a und 2b) und ob diese substantiell abnehmen, wenn die als Vermittler vermuteten Kinderkosten kontrolliert werden (Modelle 3a und 3b).

Als übergeordneter Befund des ersten Modells ist festzuhalten, dass die von den Frauen wahrgenommenen Kosten die Erklärung dominieren: Beide Kostenarten wirken sich signifikant nachteilig auf die Geburt des ersten Kindes aus. Dass vor allem die Opportunitätenkosten der Frau die Familiengründung negativ beeinflussen $(\operatorname{Exp}(B)=0,73$; $\mathrm{p} \leq 0,01$ ) und nicht die des Mannes, stärkt die Annahme der NHE, dass diese Kosten hauptsächlich im Zusammenhang mit der Frau entstehen. Partiell finden sich auch Hinweise für die Richtigkeit der Einkommenshypothese: Es zeigt sich wenigstens ein negativer Effekt der von der Frau eingeschätzten direkten Kinderkosten auf die Familiengründungsneigung des Paares $(\operatorname{Exp}(B)=0,74 ; p \leq 0,05)$. Dass die von Männern mit Kindern in Verbindung gebrachten direkten Kosten ohne Einfluss auf die Wahrscheinlichkeit der Geburt eines ersten gemeinsamen Kindes bleiben, ist nicht nur mit Blick auf eine Bewertung der NHE ein höchst interessanter Befund - vorausgesetzt dahinter verbirgt sich keine, vor allem Männer betreffende fehlerhafte Kostenerfassung.

Tabelle 2: Determinanten des Übergangs zur Elternschaft

\begin{tabular}{|c|c|c|c|c|c|}
\hline \multirow{2}{*}{ Prädiktoren } & \multicolumn{5}{|c|}{ Exp(B) + Signifikanz } \\
\hline & 1 & 2a & $2 b$ & 3a & 3b \\
\hline Direkte Kosten Mann & 1,22 & & 1,20 & & 1,12 \\
\hline Opportunitätenkosten Mann & 0,87 & & 0,81 & & 0,83 \\
\hline Bildung Mann & & $0,78^{*}$ & $0,76^{* *}$ & & \\
\hline Direkte Kosten Frau & 0,74 * & & $0,65^{\star *}$ & & $0,75^{*}$ \\
\hline Opportunitätenkosten Frau & $0,73^{* *}$ & & 0,81 & & $0,79^{\star *}$ \\
\hline Bildung Frau & & & & $0,73^{\star *}$ & $0,79^{*}$ \\
\hline Pseudo- $R^{2}$ & 0,30 & 0,21 & 0,31 & 0,29 & 0,31 \\
\hline N Gesamt (Zensiert) & & & $206(30$ & & \\
\hline
\end{tabular}

Quelle: PAIRFAM Vorstudie 2006. Anmerkung: Ereignisanalyse nach generalisiertem log-logistischen Modell, Schätzung des Risikoparameters; ${ }^{*} \mathrm{p} \leq 0,05 ; * * \mathrm{p} \leq 0,01$.

Die nächsten Modelle liefern weitere aufschlussreiche Ergebnisse. Im mittleren Teil der Tabelle 2 wird die Prüfung der Einkommenshypothese vertieft. Gänzlich entgegen der 
Erwartung tritt ein negativer Bildungseffekt des Mannes auf die Familiengründung hervor (Beta $=0,78 ; p \leq 0,05)$. Gemäß der Einkommenshypothese wäre zu erwarten gewesen, dass mit steigendem Bildungsniveau des Mannes (und damit zunehmendem Einkommen des Paarhaushaltes) die Wahrscheinlichkeit der Kindergeburt steigt. Angesichts dieses Befundes erübrigt sich die Frage nach der Vermittlung dieses Effektes. Dennoch kann das Modell $2 b$ ergänzen, dass der negative Einfluss, der von der Frau wahrgenommenen direkten Kosten unter Konstanthaltung der Bildung ihres Mannes erhalten bleibt bzw. nunmehr sogar hoch signifikant ist $(\operatorname{Exp}(B)=0,65 ; p \leq 0,01)$. Dieses Ergebnis impliziert, dass die von der Frau wahrgenommenen direkten Kinderkosten unabhängig vom Bildungshintergrund ihres Partners gegen eine Familiengründung sprechen. Unter der Annahme einer korrekten Indikatorbildung ist das als bemerkenswertes Indiz gegen die Einkommenshypothese $\mathrm{zu}$ interpretieren. Nur etwas günstiger gestaltet sich die Befundlage mit Blick auf die Opportunitätenkostenhypothese (Modelle 3). Zwar bestätigt sich zunächst ein erwarteter negativer Bildungseffekt: Mit steigendem Bildungsniveau der Frau nimmt die Wahrscheinlichkeit der Familiengründung ab (Beta $=0,73 ; p \leq 0,05$ ). Das Modell $3 b$ verweist dann aber auf eine recht eingeschränkte Vermittlung dieses Effektes, da er unter Kontrolle der Kinderkosten nur wenig an Stärke verliert (Beta $=0,79 ; p \leq 0,05$ ), bei gleichzeitig hoch signifikantem, negativen Einfluss der Opportunitätenkosten der Frau (Beta $=0,79 ; \mathrm{p} \leq 0,01)$.

Zusammenfassend lassen sich also verschiedene eigenständige Effekte auf die Familiengründung festhalten - einerseits ausgehend von der Bildung beider Partner und andererseits ausgehend von den kindbezogenen Kosten, die von der Frau wahrgenommen werden. Überraschend ist die scheinbar weitgehende Unabhängigkeit beider Konzepte mit Blick auf die Erklärung des generativen Verhaltens. Es ist zu vermuten, dass diese zumindest teilweise auf eine unbefriedigende Erfassung des Bildungskonzeptes zurückgeht: Mit der Beschränkung auf die Schulbildung scheint die erwerbs- und einkommensbezogene Komponente dieses Konzeptes zu Gunsten alternativer Informationsanteile wie Lebenskonzepte, Einstellungen oder Ansprüche in den Hintergrund zu treten. Das erweist sich für die Prüfung der einkommensbasierten Argumentation der NHE als nachteilig und entsprechend bleiben aus diesen Analysen ableitbare Zweifel an der Gültigkeit der zentralen Thesen der NHE sehr vage. Aber selbst wenn es zutreffend ist, dass die hier gefundenen Bildungseffekte nur bedingt Einkommenseffekte widerspiegeln, würde das auf eine Schwäche der NHE hinweisen, da diese nicht vorsieht, dass mit zunehmendem Bildungsniveau die Familiengründungsneigung auch jenseits eines möglichen Einkommenseffektes abnimmt.

\section{Zusammenfassung und Diskussion}

Trotz offensichtlicher Nachteile des Stichprobendesigns und der Indikatorbildung bot sich angesichts der von beiden Partnern subjektiv eingeschätzten Kinderkosten die Möglichkeit, Argumenten der NHE empirisch nachzugehen, die bisher wenig Beachtung gefunden haben. Und in der Tat empfehlen die Ergebnisse, Prüfungen in dieser Richtung zu vertiefen, denn auch wenn einige der gefundenen Zusammenhänge im Sinne der NHE ausfallen, lassen sich ihre zentralen Annahmen empirisch kaum fundieren. Die noch stärkste Bestätigung erfährt die Opportunitätenkostenhypothese. Gleichwohl fällt der für die Frau 
negative, über ihre Opportunitätenkosten vermittelte Bildungseffekt auf die Familiengründung sehr beschränkt aus. Auch für die oft stiefmütterlich behandelte Einkommenshypothese finden sich zwar zunächst insofern Anhaltspunkte, als die auf die Familiengründung negativ wirkenden direkten Kostenwahrnehmungen seitens der Frau mit steigendem Bildungsniveau ihres Partners zurückgehen. Allerdings können gerade die letzten Analysen die vermittelnde Position der direkten Kinderkosten zwischen Bildung und generativem Verhalten nicht bestätigen. Gegen die Einkommenshypothese spricht zudem, dass das Bildungsniveau teilweise positiv mit den direkten Kinderkosten zusammenhängt und auf diese Weise auch die Wahrscheinlichkeit einer Erstgeburt herabsetzt. Dieser Wirkungspfad existiert innerhalb der NHE nicht. Würde er sich in späteren Analysen bestätigen, dann wäre eine nicht unerhebliche Schlussfolgerung, dass die Geburtenneigung hoch gebildeter Frauen in zweifacher Hinsicht gemindert wird - einmal durch die bekannte Zunahme ihrer Opportunitätenkosten und darüber hinaus durch einen Anstieg der von ihnen wahrgenommenen direkten Kinderkosten. Zudem deuten die Ergebnisse zumindest vage an, dass das Bildungsniveau nur bedingt als Indikator für Kinderkosten fungiert bzw. nur eingeschränkt über die Kinderkosten auf das generative Verhalten Einfluss nimmt.

Ebenfalls als erwartungskonträr erweist sich die ausschließliche Verhaltensrelevanz der Kostenwahrnehmung der Frau. Dieses Ergebnis schwächt die von der NHE unterstellte Annahme gemeinsam getroffener Haushaltsentscheidungen nach dem Grundsatz der maximalen Haushaltsnutzenproduktion erheblich, der zufolge die von beiden Partnern wahrgenommenen Kosten in vergleichbarem Umfang die Familiengründung determinieren sollten. Somit entsteht eine Erklärungslücke, die zu schließen es bedarf. Vielversprechend hierfür scheint eine weiterführende Auswertung getrennt nach der partnerschaftlichen Bildungskonstellation, um so festzustellen, ob bereits vorgelegte, verhandlungstheoretische Erweiterungen der NHE (u.a. Ott 1989) zu realistischeren Annahmen und besseren Erklärungen führen. Wird das durchschnittlich hohe Bildungsniveau der in diesem Datensatz enthaltenen Frauen in Erinnerung gerufen, so könnte man ihnen in diesem Sinne eine grundsätzlich sehr gute Verhandlungsposition gegenüber ihren Partnern unterstellen. Das wiederum sollte sie dazu befähigen, ihre Interessen bei der generativen Entscheidung besser durchzusetzen. Leider konnte in Anbetracht der geringen Fallzahl dieser Überlegung empirisch nicht nachgegangen werden.

Eine praktische Konsequenz, die aus dem Befund der ausschließlichen Erklärungskraft weiblicher Kosten resultieren könnte, wäre die lange Zeit übliche und nunmehr empirisch legitimierte Beschränkung auf die Perspektive der Frau. Entkräftet wird ein solcher Vorschlag jedoch dadurch, dass auch die Bildung des Mannes die Familiengründung jenseits ökonomischer Kinderkosten zu beeinflussen scheint. Denkbare Begründungen hierfür wären nicht ohne weiteres aus der NHE ableitbar. Das sich dadurch abzeichnende Erklärungsdefizit könnte möglicherweise durch die Berücksichtigung von Nutzen bzw. Vorteilen, die mit Kindern einhergehen, beseitigt werden. Becker selbst bringt mit Kindern sowohl einen Konsum- als auch einen Produktionsnutzen in Verbindung (1960: 210), verfolgt beide aber nicht weiter bzw. behauptet, sie seien konstant. In der Value of Children-Forschung werden qualitativ unterschiedliche Nutzenaspekte von Kindern nicht nur theoretisch hergeleitet, sondern auch systematisch mit den individuell wahrgenommenen Handlungsbedingungen und dem generativen Verhalten verbunden (Nauck 2001). Ein steigendes Bildungsniveau impliziert in diesem Erklärungszusammenhang einen allge- 
meinen Rückgang des Nutzens von Kindern - ein Zusammenhang der dazu beitragen könnte, negative Bildungseffekte auf die Fertilität weiter aufzulösen.

Schließlich sind trotz der benannten Vorteile auch Beschränkungen der verwendeten Daten nicht von der Hand zu weisen, die die Aussagekraft der Befunde schmälern. Wie bereits erwähnt, ist die Stichprobe sehr klein und auf die städtische Bevölkerung begrenzt, was zumindest eine Wiederholung dieser Analysen auf breiter Datenbasis empfiehlt. An verschiedenen Stellen der Untersuchung deutet sich eine eingeschränkte Brauchbarkeit des hier verwendeten Bildungsindikators zur Abbildung einkommensbezogener Ressourcen an. Deshalb können daran geknüpfte Befunde lediglich als Arbeitshypothesen für weiterführende Untersuchungen dienen. Außerdem stehen auf Grund des Querschnittsdesigns der Stichprobe die Kostenwahrnehmungen von Befragten mit Kind(ern) lediglich für die Zeit nach der Erstgeburt zur Verfügung. Das beeinträchtigt die Gültigkeit ihrer Messung insbesondere dann, wenn Abhängigkeiten von der jeweiligen Lebens- bzw. Familienphase bestehen, in der sich der Befragte zum Befragungszeitpunkt befindet. Zumindest lassen sich weder theoretische Anhaltspunkte noch empirische Befunde für systematische oder substantielle Verzerrungen dieser Art finden, weshalb maximal eine Unterschätzung der aufgefundenen Effekte geschlussfolgert wird. Dennoch ist evident: Nur „panel data in which perceived costs and rewards of children are measured before the actual behaviour occurs can be used to study the causal impact of costs and rewards on actual decisions on childbearing" (Liefbroer 2005: 368). Dementsprechend können die aus den Analysen gezogenen Schlussfolgerungen nur als erste Indizien betrachtet werden, die unter Verwendung prospektiv gewonnener Längsschnittdaten größeren Umfangs zu replizieren sind. Das aber bedeutet, dass entsprechende Daten zunächst erhoben werden, denn diesbezüglich ist für die Forschung im deutschsprachigen Raum erheblicher Nachholbedarf festzustellen.

\section{Literatur}

Arnold, F. et al. (1975). The value of children: A cross-national study. Honolulu: East-West Center.

Bagozzi, R. P. \& van Loo, M. F. (1978). Fertility as consumption: Theories from the behavioral science. Journal of Consumer Research, 4, pp. 199-228.

Baron, R. M. \& Kenny, D. A. (1986). The moderator-mediator variable distinction in social psychological research: Conceptual, strategic, and statistical considerations. Journal of Personality and Social Psychology, 6, pp. 1173-1182.

Becker, G. S. (1960). An economic analysis of fertility. In: National Bureau of Economic Reasearch (Ed.), Demographic and economic change in developed countries. Princeton: Princeton University Press, pp. 209-231.

Becker, G. S. (1965). A theory of the allocation of time. The Economic Journal, 75, pp. 493-517.

Becker, G. S. (1981). A treatise on the family. Cambridge: Harvard University Press.

Becker, G. S. \& Lewis, H. G. (1973). On the interaction between the quantity and quality of children. Journal of Political Economy, 2, pp. 279-288.

Blossfeld, H.-P. \& Huinink, J. (1989). Die Verbesserung der Bildungs- und Berufschancen von Frauen und ihr Einfluss auf die Familienbildung. Zeitschrift für Bevölkerungswissenschaft, 15. S. 383-404.

Blossfeld, H.-P. \& Huinink, J. (1991). Human capital investments or norms of role transition? How women's schooling and career affect the process of family formation? American Journal of Sociology, 97, pp. 143-168. 
Blossfeld, H.-P. \& Jänichen, U. (1990). Bildungsexpansion und Familienbildung. Wie wirkt sich die Höherqualifikation der Frauen auf ihre Neigung zu heiraten und Kinder zu bekommen aus? Soziale Welt, 41, S. 454-476.

Brentano, L. (1910). The doctrine of Malthus and the increase of population during the last decades. Economic Journal, 20, pp. 371-393.

Brüderl, J. \& Diekmann, A. (1995). The log-logistic rate model: Two generalizations with an application to demographic data. Sociological Methods \& Research, 2, pp. 158-196.

Brüderl, J. \& Klein, T. (1991). Bildung und Familiengründung: Institutionen- versus Niveaueffekt. Zeitschrift für Bevölkerungswissenschaft, 3, S. 323-335.

Brüderl, J. \& Klein, T. (1993). Bildung- und Familiengründungsprozeß deutscher Frauen: Humankapitalund Institutioneneffekt. In: A. Diekmann \& S. Weick (Hrsg.), Der Familienzyklus als sozialer Prozess. Berlin: Duncker und Humblot, S. 184-215.

Butz, W. P. \& Ward, M. P. (1979). The emergence of countercyclical U.S. fertility. American Economic Review, 69, pp. 318-328.

Cain, G. G. \& Weiniger, A. (1973). Economic determinants of fertility. Results from cross-sectional aggregate. Demography, 10, pp. 205-223.

Conger, D. J. \& Campbell, J. M. (1978). Simultaneity in the birth rate equation: The effects of education, labor force particpiation, income, and health. Econometrica, 46, pp. 631-641.

de Tray, D. N. (1973). Child quality and the demand for children. Journal of Political Economy, 81, pp. 70-98.

Duschek, K.-J. \& Wirth, H. (2005). Kinderlosigkeit von Frauen im Spiegel des Mikrozensus. Eine Kohortenanalyse der Mikrozensen 1987-2003. Wirtschaft und Statistik, 8, S. 800-820.

Eckhard, J. \& Klein, T. (2007). Die Motivation zur Elternschaft. Unterschiede zwischen Männern und Frauen. In D. Konietzka \& M. Kreyenfeld (Hrsg.), Ein Leben ohne Kinder. Kinderlosigkeit in Deutschland. Wiesbaden: VS Verlag, S. 275-294.

Fawcett, J. T. (1978). The value and cost of the first child. In: W. B. Miller \& L. F. Newman (Eds), The first child and family formation. Chapel Hill: Carolina Population Center, pp. 244-265.

Hashimoto, M. (1974). Economics of postwar fertility in Japan. In T. W. Schultz (Ed.), Economics of the family. Chicago: University Press, pp. 225-254.

Kashy, D. A. \& Kenny, D. A. (2000). The analysis of data from dyads and groups. In: H. T. Reis \& C. M. Judd (Eds), Handbook of research methods in social psychology. New York: Cambridge University Press, pp. 451-477.

Klaus, D. (2008). Sozialer Wandel und Geburtenrückgang in der Türkei. Wiesbaden: VS Verlag.

Klein, T. (2003). Die Geburt von Kindern in paarbezogener Perspektive. Zeitschrift für Soziologie, 32, S. 506-527.

Klein, T. \& Eckhard, J. (2005). Bildungsbezogene Unterschiede des Kinderwunschs und des generativen Verhaltens. Eine kritische Analyse der Opportunitätskostenhypothese. In: A. Steinbach (Hrsg.), Generatives Verhalten und Generationenbeziehungen. Wiesbaden: VS Verlag, S. 151-176.

Klein, T. \& Lauterbach, W. (1994). Bildungseinflüsse auf Heirat, die Geburt des ersten Kindes und die Erwerbsunterbrechung von Frauen. Eine empirische Analyse familienökonomischer Erklärungsmuster. Kölner Zeitschrift für Soziologie und Sozialpsychologie, 46, S. 278-298.

Kreyenfeld, M. \& Konietzka, D. (2007). Kinderlosigkeit in Deutschland - theoretische Probleme und empirische Ergebnisse. In: D. Konietzka \& M. Kreyenfeld (Hrsg.), Ein Leben ohne Kinder. Wiesbaden: VS Verlag. S. 11-41.

Kurz, K. (2005). Die Familiengründung von Männern im Partnerschaftskontext. In: A. Tölke \& K. Hank (Hrsg.), Männer - Das ,,vernachlässigte“ Geschlecht, Wiesbaden: VS Verlag, S. 178-197 (Sonderheft 4 der Zeitschrift für Familienforschung).

Leibenstein, H. (1957). Economic backwardness and economic growth. New York: Wiley \& Sons, Inc.

Leibenstein, H. (1974). An interpretation of the economic theory of fertility: Promising path or blind alley? Journal of Economic Literature, 12, pp. 457-479.

Liefbroer, A. C. (2005). The impact of perceived costs and rewards of childbearing on entry into parenthood: Evidence from a panel study. European Journal of Population, 21, pp. 367-391. 
Mincer, J. (1963). Market prices, opportunity costs, and income effects. In: C. F. Christ (Ed.), Measurements in economics. Stanford: University Press, pp. 67-82.

Nauck, B. (2001). Value of children: Eine spezielle Handlungstheorie des generativen Verhaltens und von Generationenbeziehungen im interkulturellen Vergleich. Kölner Zeitschrift für Soziologie und Sozialpsychologie, 53, S. 407-435.

Nauck, B. (2007). Value of children and the framing of fertility: Results from a cross-cultural comparative survey in 10 societies. European Sociological Review, 23, pp. 615 - 629.

Ott, N. (1989). Familienbildung und familiale Entscheidungsfindung aus verhandlungstheoretischer Sicht. In: G. Wagner, N. Ott \& H.-J. Hoffmann-Nowotny (Hrsg.), Familienbildung und Erwerbstätigkeit im demographischen Wandel, Berlin: Springer, S. 97-116.

Scanzoni, J. (1975). Sex roles, life styles and childbearing. New York: Free Press.

Schaeper, H. (2007). Familiengründung von Hochschulabsolventinnen. Eine empirische Untersuchung verschiedener Examenskohorten. In: D. Konietzka \& M. Kreyenfeld (Hrsg.), Ein Leben ohne Kinder. Wiesbaden: VS Verlag, S. 137-166.

Timm, A. (2006). Die Veränderung des Heirats- und Fertilitätsverhaltens im Zuge der Bildungsexpansion. Eine Längsschnittanalyse für West- und Ostdeutschland. In: A. Hadjar \& R. Becker (Hrsg.), Die Bildungsexpansion. Erwartete und unerwartete Folgen. Wiesbaden: VS Verlag, S. 277-309.

Trommsdorff, G. \& Nauck, B. (2005) (Eds). The value of children in cross-cultural perspective. Case studies from eight societies. Lengerich: Pabst Science.

Turchi, B. A. (1975). Microeconomic theories of fertility: A critique. Social Forces, 1, pp. 107-25.

van de Kaa, D. J. (1987). Europe's second demographic transition. Population Bulletin, 1, pp. 1-59.

Willis, R. J. (1973). A new approach to the economic theory of fertility behaviour. Journal of Political Economy, Supplement, 81, pp. 14-69.

Wirth, H. (2007). Kinderlosigkeit von hochqualifizierten Frauen und Männern im Paarkontext - Eine Folge von Bildungshomogamie? In: D. Konietzka \& M. Kreyenfeld (Hrsg.), Ein Leben ohne Kinder. Wiesbaden: VS Verlag. S. 167-200.

Eingereicht am/Submitted on: 17. 06. 2008

Angenommen am/Accepted on: 07. 09. 2009

Anschrift der Autorin/Address of the author:

Dr. Daniela Klaus

Technische Universität Chemnitz

Institut für Soziologie

Thüringer Weg 9

D-09107 Chemnitz

E-mail: daniela.klaus@soziologie.tu-chemnitz.de 